\title{
A NEW METHOD OF DETERMINATION OF COLLAGEN CONJUGATED WITH KERATIN
}

\author{
Marta Safandowska, Krystyna Pietrucha
}

Department of Material and Commodity Sciences and Textile Metrology, Lodz University of Technology, Zeromskiego 116, 90-924 Lodz, Poland

E-mail: marta.safandowska@wp.pl

\begin{abstract}
:
The paper describes the possibility of using Sirius red dye for the determination of collagen conjugated with keratin of wool. Sirius red assay was shown to be feasible for collagen detection, which was enzymatically coupled onto wool fibers and woven fabric. The effectiveness of combination of keratin protein with collagen was evaluated.
\end{abstract}

\section{Keywords:}

Collagen, Sirius red, keratin

\section{Introduction}

Collagen is the major component of the extracellular matrix (ECM) and is the most abundant mammalian protein accounting for about $20-30 \%$ of the total body proteins. The unique biocompatibility due to its biological characteristics, such as biodegradability and weak antigenicity, made collagen the primary resource in medical applications [1]. Collagen can also be used to modify the surface of various types of synthetic polymers. For example, coating polypropylene meshes with collagen results in their improved bio- and cytocompatibility; modification of polyester vascular prosthesis by collagen increases their leak-proof properties [2-4]. Furthermore, collagen displayed bacteriostatic properties against Staphylococcus epidermidis and $\beta$-hemolytic Streptococcus [5], and therefore it can impart antibacterial properties to natural textile fibers [6].

A multitude of applications of collagen indicates that it is very appropriate to use a rapid and precise method for its determination. Many physicochemical methods, such as X-ray photoelectron spectroscopy (XPS), chromogenic reactions (hydroxyproline assay, amine dyes: ninhydrin or Rhodamine B isothiocyanate), molecular weight comparisons (gel electrophoresis, chromatography), radioactive procedures (radioactive labeling of proline) and immunological reactions (enzymeimmunoassays using specific antibodies) are used to estimate the amount of collagen [2,3]. The most precise and specific method of collagen determination is based on the quantitation of its hydroxyproline content; unfortunately, it is a complex and time consuming procedure. Sirius red staining seems to be an excellent alternative technique for quantitative or qualitative measurement of collagen. It is a fast and nondestructive method, which has been used for histological staining of collagen in tissue sections from many years [7].

The goal of this study is to elaborate a simple and accurate method of collagen examination. In this work, the method of Sirius red staining was applied to assay collagen conjugated with keratin of wool. The application of Sirius red dye for the detection of collagen on keratin substrates has not been reported previously. The capability of tyrosinase to catalyze the oxidation of tyrosine residues of keratin and for coating collagen on wool materials has to be assessed.

\section{Materials and methods}

\subsection{Materials}

Wool fibers and woven fabric (twill weave) were chosen for preparation of the samples. Fibers from sheep wool was cleaned by Soxhlet extraction using dichloromethane to remove fatty matters ( $t=14 \mathrm{~h}, 6$ transfers to $1 \mathrm{~h}$ ). Collagen type I was prepared from fresh skin of silver carp and supplied by AAG Sp. z o.o. (Poland).

Tyrosinase from mushroom (EC 1.14.18.1, $\geq 1000$ unit/mg solid) and Sirius red F3BA were purchased from Sigma-Aldrich (Sigma, St. Louis, MO, USA). All other chemicals of analytical grade were obtained from POCh-Gliwice (Poland).

\subsection{Preparation of samples}

Samples of wool fibers and fabric were modified with enzyme and collagen in accordance with a method [6]. For this purpose, $0.5 \mathrm{~g}$ wool was placed in $50 \mathrm{ml} 0.1 \mathrm{M}$ phosphate buffer $(\mathrm{pH}=6.5)$ containing tyrosinase $(2000 \mathrm{U} / \mathrm{g})$. In addition, ascorbic acid solution at $0.42 \mathrm{mg} / \mathrm{ml}$ was added. After $1 \mathrm{~h}$, collagen solution in $1 \% \mathrm{CH}_{3} \mathrm{COOH}$ was added to the buffer/wool incubation mixture in a final concentration of $2 \mathrm{mg} / \mathrm{ml}$. Incubation was continued at $25^{\circ} \mathrm{C}$ for $24 \mathrm{~h}$. To terminate the enzymatic reaction, the $\mathrm{pH}$ was raised to $\mathrm{pH} 9$ using $0.1 \mathrm{M} \mathrm{KOH}$. Then, the sample was rinsed in distilled water and $1 \% \mathrm{CH}_{3} \mathrm{COOH}$, and air-dried.

\subsection{Characterization of the products}

Color measurements. In order to determine the enzymecatalytic modification effect on the wool-derived keratin, the reflectance measurements were evaluated by using Datacolor 
Int. Spectraflash 500 spectrophotometer with dataMaster software. Results were expressed by the CIE whiteness (W) according to the ISO 105-J02:1999 method and CIELab color values and color difference $(\Delta \mathrm{E})$ at $\mathrm{D} 65 / 10^{\circ}$. The degree of whiteness $(\mathrm{W})$ was calculated using the following equation:

$$
W=Y+800(0.3138-x)+1700(0.3310-y)
$$

where:

$W$ is the whiteness; $Y$ the trichromatic component of the sample; $x, y$ the chromaticity coordinates of the samples, 0.3138 and 0.3310 the chromaticity coordinates $x$ and $y$ respectively for the perfect light scattered.

The color difference - $\Delta \mathrm{E}$ - was calculated according to the formula of Commission Internationale de l'Eclairage - CIE Lab in relation to an unmodified sample:

$$
\Delta E^{*}=\sqrt{\left(\Delta L^{*}\right)^{2}+\left(\Delta a^{*}\right)^{2}+\left(\Delta b^{*}\right)^{2}}
$$

where:

$\Delta E$ is the color difference expressed in CIELab units; $\Delta L$ the difference in lightness; $\Delta a$ the difference in the chromaticity coordinate, green/red axis; $\Delta b$ the difference in the chromaticity coordinate, blue/yellow axis.

Sirius red staining. The presence of collagen onto the surface layer of wool-derived keratin was evaluated by staining with Sirius red F3BA dye, in accordance with the procedure described by [7]. Tyrosinase-treated sample in the presence and absence of collagen were incubated with water solution of $0.5 \%$ Sirius red at room temperature for 30 minutes. Thereafter, the samples were washed extensively for 30 minutes in distilled water, and air-dried. The color measurements were made by sensory impairments.

\section{Results}

As revealed in Figure 1, the CIE whiteness (W-CIE D65/10) of keratin samples (wool fibers and woven fabric) treated by tyrosinase in comparison with untreated samples increased by $15 \%$ and $4 \%$, respectively. The increase in whiteness was also observed for the samples which were subjected to enzyme treatment and simultaneous coating with collagen.

The increase in the value of whiteness may be related to the fact that the tyrosine residues of keratin under reducing conditions and under the influence of the enzyme are oxidized to the corresponding quinones, which possess fluorescence properties [8]. These o-quinones may either condense with each other or react with free amino groups, resulting in the formation of covalent keratin-collagen crosslinks (Figure 2) $[8,9]$.

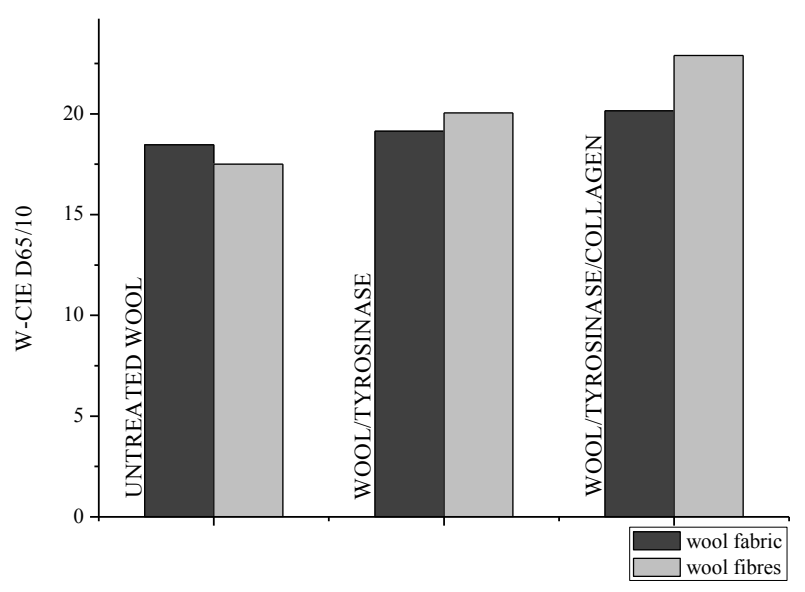

Figure 1. CIE whiteness of samples of wool-derived keratin.

As can be seen from Table 1 the enzyme treatment also had a slight impact on the color difference $(\Delta \mathrm{E})$ and color depth $(\mathrm{K} / \mathrm{S})$.

The presence of collagen on surfaces of wool was confirmed by using the staining of collagen with Sirius red F3BA. As can be seen from the pictures (Figure 3), all samples are stained; however, only those wool samples which have been enzymatically treated and simultaneously coated with collagen were characterized by a higher intensity of staining. The uncoated samples of keratin (Figure 3 A, B, D, E) did not bind the Sirius dye and gave only a weak background.

Table 1. CIELLAB color difference $(\Delta \mathrm{E})$ and color depth $(\mathrm{K} / \mathrm{S})$ of wool samples.

\begin{tabular}{|l|c|c|}
\hline \multicolumn{1}{|c|}{ Samples } & $\boldsymbol{\Delta E}$ & $\mathrm{K} / \mathbf{S}$ \\
\hline Wool fibers & - & 0,4976 \\
\hline Wool woven fabric & - & 0,5058 \\
\hline Enzyme-treated fibers & 1,58 & 0,4613 \\
\hline Enzyme-treated fabric & 0,67 & 0,4879 \\
\hline Enzyme/collagen-treated fibres & 1,98 & 0,4543 \\
\hline Enzyme/collagen-treated fabric & 1,24 & 0,4756 \\
\hline
\end{tabular}<smiles>Oc1ccc(C(Br)(Br)Br)cc1</smiles>

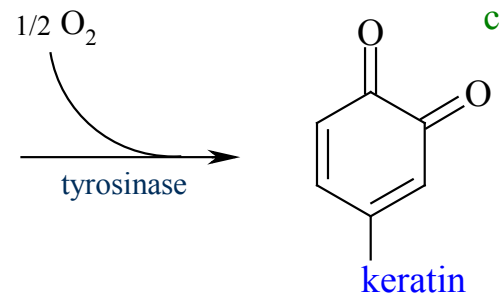<smiles>CC(C)(C)[C@@H](N)c1cc(O)c(O)cc1N[14CH3]</smiles>

Figure 2. Tyrosinase-catalyzed oxidation of tyrosine and subsequent nonenzymatic reactions of the quinone with collagen. 

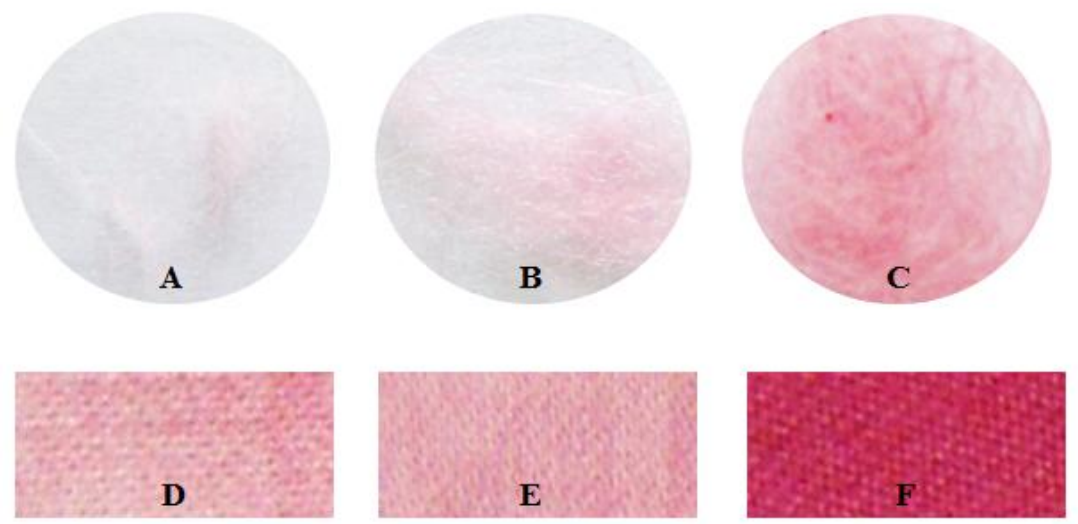

Figure 3. Surface images of wool fibers $(A, B, C)$ and woven fabric $(D, E, F)$ stained with Sirius red $(A, D)$ before enzyme treatment, $(B, E)$ after tyrosinase treatment, $(C, F)$ after tyrosinase treatment and simultaneous coating with collagen.

\section{Conclusions}

The obtained results demonstrate that Sirius red staining is a specific and simple method for the determination of collagen coated onto wool-derived keratin. Reflectance measurements showed that the whiteness of the wool samples after enzyme treatment increases, which means that tyrosinase activates the tyrosine residues in keratin to the quinone forms, which react further nonenzymatically with primary amino groups of collagen.

\section{Acknowledgment}

The work was partially supported by the National Science Centre via Grant No. DEC-2011/03/B/ST8/05867.

\section{References}

[1] Lee C.H., Singla A., Lee Y., Biomedical applications of collagen, International Journal of Pharmaceutics 221, 1-22, 2001.

[2] Zhu Y.; Gao C.; Liu X., Shen J.: Surface modification of polycaprolactone membrane via aminolysis and biomacromolecule immobilization for promoting cytocompatibility of human endothelial cells, Biomacromolecules 2, 1312-1319, 2002.

[3] Jou C.H.; Lin S.M.; Yun L.; Hwang M.C.; Yu D.G.; Chou W.L.; Lee J.S.; Yang M.C.: Biofunctional properties of polyester fibres grafted with chitosan and collagen, Polymers for Advanced Technology 18, 235-239, 2007.

[4] Pietrucha K., New collagen implant as dural substitute, Biomaterials 12, 320-323, 1991.

[5] Carlson G. A., Dragoo J. L., Samimi B., Bruckner D. A., Bernard G. W., Hedrick M., Benhaim P., Bacteriostatic properties of biomatrices against common orthopaedic pathogens, Biochemical and biophysical Research Communications 321, 472-478, 2004.

[6] Jus S.; Kokol V.; Guebitz G.M.: Tyrosinase-Catalysed coating of wool fibers different protein-based biomaterials, Journal of Biomaterials Science 20, 253-269, 2009.

[7] Junquiera L.C.U.; Bignolas G.; Brentani R.R.: A simple and sensitive method for the quantitative estimation of collagen, Analytical Biochemistry 94, 96-99, 1979.

[8] Jus S.: Kokol V.; Guebitz G.M.: Tyrosinase-catalysed coupling of functional molecules onto protein fibres, Enzyme and Microbial Technology 42, 535-542, 2008.

[9] Thalmann C.R., Lotzbeyer T., Enzymatic cross-linking of proteins with tyrosinase, EUR Food Res Technol 214, 276-281, 2002. 